\title{
Wide-Band Tuning of the Gain Spectra of One-Pump Fiber Optical Parametric Amplifiers
}

\author{
Michel E. Marhic, Senior Member, IEEE, Kenneth Kin-Yip Wong, Member, IEEE, and \\ Leonid G. Kazovsky, Fellow, IEEE
}

\begin{abstract}
By suitably choosing the fiber properties, and by tuning the pump wavelength near the fiber zero-dispersion wavelength, one can in principle generate a wide variety of one-pump fiber optical parametric amplifier gain spectra. These can range from a very wide single region to two symmetric narrow gain regions far away from the pump. We have experimentally verified these predictions. With a highly nonlinear fiber, we have inferred the existence of gain over a single 400-nm region and measured a maximum on-off gain of $65 \mathrm{~dB}$. With a common dispersion-shifted fiber, we have obtained tunable gain regions less than $1 \mathrm{~nm}$ wide, up to $200 \mathrm{~nm}$ from the pump; we have also shifted these by several nanometers by lowering the fiber temperature to $0^{\circ} \mathrm{C}$.
\end{abstract}

Index Terms-Parametric amplifiers, tunable amplifiers, tunable filters.

\section{INTRODUCTION}

$\mathbf{T}$ HE DETAILED shape of the gain spectra obtainable with one-pump fiber optical parametric amplifiers (OPAs) was first investigated in [1]. It was shown that the spectra are symmetric in the frequency domain about the pump frequency and that their shapes are primarily determined by the even-order dispersion coefficients $\beta_{2}$ and $\beta_{4}$. Since $\beta_{2}$ can be adjusted by tuning the pump wavelength $\lambda_{p}$ near the zero-dispersion wavelength $\lambda_{0}$, one can in principle optimize gain spectra in this manner. The emphasis in [1] was to obtain a single wide gain region, and it was predicted that a gain bandwidth of the order of $100 \mathrm{~nm}$ could be obtained with common dispersion-shifted fiber (DSF), with a pump power of a few watts. Early measurements verified the theory over a 35-nm interval, limited only by the tuning range of the signal laser [1]. The advent of highly nonlinear fibers (HNLFs), with a nonlinearity coefficient $\gamma$ approximately ten times larger than that of DSF, promised even larger gain bandwidths. A 200-nm bandwidth was then demonstrated [2], and $360 \mathrm{~nm}$ has recently been achieved [3].

In other theoretical work [4], it was shown that if $\left|\lambda_{p}-\lambda_{0}\right|$ is increased beyond the value necessary for obtaining a single wide gain region, the spectrum breaks into two symmetric regions, which become narrower as they move away from $\lambda_{p}$;

Manuscript received January 12, 2004; revised May 19, 2004. This work was supported by the National Science Foundation under Grant ANI-0 123441.

M. E. Marhic and L. G. Kazovsky are with the Department of Electrical Engineering, Stanford University, Stanford, CA 94305 USA (e-mail: marhic@wdm.stanford.edu).

K. K.-Y. Wong was with the Department of Electrical Engineering, Stanford University, Stanford, CA 94305 USA. He is now with the Department of Electrical and Electronics Engineering, University of Hong Kong, Hong Kong, China.

Digital Object Identifier 10.1109/JSTQE.2004.835298 there is also a lower gain region around $\lambda_{p}$, which is less interesting. The existence of these gain regions suggests new applications. If they are about $1 \mathrm{~nm}$ wide, they could be used for making narrow-band filters, with gain, potentially tunable over tens of nanometers or more; these might find applications in optical receivers. If they are tens of nanometers wide, they could be used for making amplifiers in regions such as the $S$-band or the $O$-band, using convenient pumps in the $C$-band. Such narrow gain regions far from the pump were observed in early OPA work in standard single-mode fibers [5], [6], but they were not tuned over wide regions.

In this work we investigate, for the first time to our knowledge, the evolution of the gain spectra of one-pump fiber OPAs over their complete range, going from a single wide gain region to two symmetric narrow gain regions far away from the pump. We first present the relevant theory, and then present experimental results for DSF and HNLF. The results show important differences between the two types of fibers; in particular, we show that the longitudinal variations of $\lambda_{0}$ in HNLF are about two orders of magnitude worse than in DSF, which poses a significant problem for OPA design. We also measured the gain spectra at two different temperatures, illustrating the need for temperature control in some applications.

\section{THEORY}

\section{A. Basic Considerations}

Consider a fiber OPA with a single pump, a signal, and an idler, with angular frequencies $\omega_{p}, \omega_{s}$, and $\omega_{i}$, satisfying $2 \omega_{p}=$ $\omega_{s}+\omega_{i}$. We assume that the three waves remain in the same state of linear polarization over the entire fiber. Substantial gain can be obtained if $-4 \gamma P_{0}<\Delta \beta<0$, where $\gamma$ is the fiber nonlinear coefficient, $P_{0}$ is the pump power, and $\Delta \beta=\beta_{s}+\beta_{i}-2 \beta_{p}$ is the mismatch between the propagation constant $\beta_{s}, \beta_{i}$, and $\beta_{p}$. Hence, the edges of the gain region(s) correspond to $\Delta \beta=0$ and $\Delta \beta=-4 \gamma P_{0}$. Following [1], we define the width of a gain region as the distance between these two points.

Maximum gain is obtained when $\Delta \beta=-2 \gamma P_{0}$. Then the signal-to-idler power conversion efficiency is simply given by

$$
G_{i, \max }=\sinh ^{2}\left(\gamma P_{0} L\right) .
$$

The shape of the gain spectrum is determined by the fiber dispersion properties. We analyze the location and width of the gain regions by using a model for $\Delta \beta$ containing only the second and fourth orders of dispersion [1]. In terms of $\Delta \omega_{s}=\omega_{s}-\omega_{p}$

$$
\Delta \beta=\beta_{2}\left(\Delta \omega_{s}\right)^{2}+\beta_{4}\left(\Delta \omega_{s}\right)^{4} / 12
$$




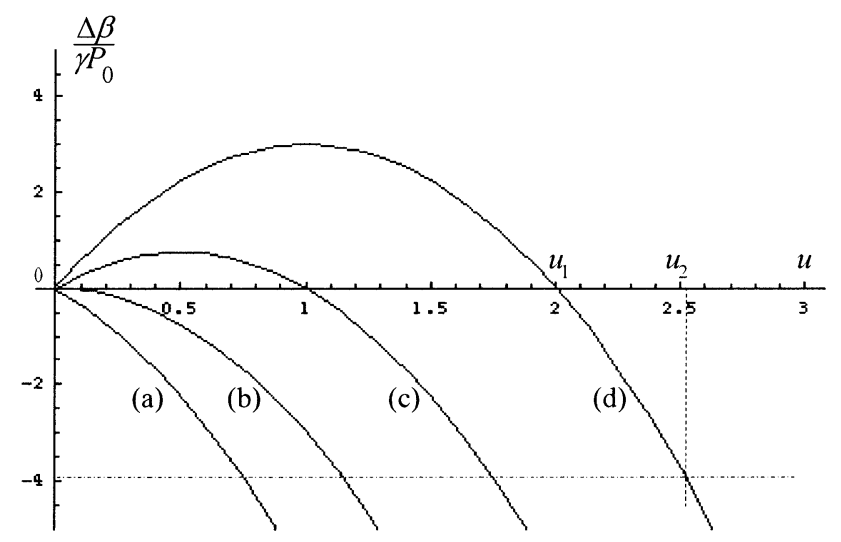

Fig. 1. Graph of $\Delta \beta / \gamma P_{0}$ versus $u=\left(\Delta \omega_{s}\right)^{2}$, for various values of $\beta_{2} . \beta_{4}$ is assumed to be negative. The units of $u$ are arbitrary. (a) $\beta_{2}<0$. (b) $\beta_{2}=0$. (c) and (d) $\beta_{2}>0$.

where $\beta_{m}$ is the $m$ th derivative of $\beta(\omega)$ with respect to $\omega$, evaluated at $\omega_{p}$.

For a given fiber, we can vary $\beta_{2}$ over a large range by tuning $\omega_{p}$ near $\omega_{0}$, because we have approximately $\beta_{2}=\beta_{3}\left(\omega_{p}-\right.$ $\left.\omega_{0}\right)$. On the other hand, $\beta_{4}$ can be assumed to remain constant; in the following, we assume that $\beta_{4}<0$, as this is the case for the fibers used in our experiments. Fig. 1 shows graphs of $\Delta \beta / \gamma P_{0}$ versus $u=\left(\Delta \omega_{s}\right)^{2}$. They are parabolas with different slopes at the origin equal to $\beta_{2}$, and the same curvature at their maxima, equal to $\beta_{4} / 6$. It can readily be seen that the widest gain spectrum (consisting of a single gain region) is obtained for $\beta_{2}=0$. Its half-width is

$$
\Delta \omega_{4}=2\left|\frac{3 \gamma P_{0}}{\beta_{4}}\right|^{1 / 4}
$$

The shape of the gain spectra changes rapidly as $\beta_{2}$ passes through 0 , but becomes fairly stable on either side. If $\beta_{2}$ becomes increasingly negative (anomalous dispersion), the width of that single region become smaller. If $\beta_{2}$ becomes increasingly positive (normal dispersion), the gain region breaks into two pieces: a central region whose width shrinks, and a region which moves away from $u=0$, and whose width shrinks in the process [case (d)]. By adjusting $\omega_{p}$ we can in principle position this region at will. In the following we will investigate in detail the main features of the gain spectrum corresponding to case (d), as it may lead to applications heretofore not envisioned for fiber OPAs.

In examples, we are going to consider OPAs similar to those that we used in the experiments. We consider the following two types of OPAs:

1) fiber: Corning DSF; $L=200 \mathrm{~m} ; P_{0}=12 \mathrm{~W}$; $\gamma=2.3 \mathrm{~W}^{-1} \mathrm{~km}^{-1} ; \lambda_{0}=1542.3 \mathrm{~nm} ; \beta_{3}=$ $1.14 \times 10^{-40} \mathrm{~s}^{3} \mathrm{~m}^{-1} ; \beta_{4}=-5 \times 10^{-55} \mathrm{~s}^{4} \mathrm{~m}^{-1}$.

2) fiber: Furukawa HNLF; $L=30 \mathrm{~m} ; P_{0}=20$ $\mathrm{W} ; \gamma=18 \mathrm{~W}^{-1} \mathrm{~km}^{-1} ; \lambda_{0}=1556 \mathrm{~nm} ; \beta_{3}=$ $0.49 \times 10^{-40} \mathrm{~s}^{3} \mathrm{~m}^{-1} ; \beta_{4}=-5.8 \times 10^{-56} \mathrm{~s}^{4} \mathrm{~m}^{-1}$.

\section{B. Shape of the Narrow Gain Spectrum}

It is interesting to investigate in some detail the shape of the narrow gain spectrum obtained for large $\beta_{2}$ (pump in the normal

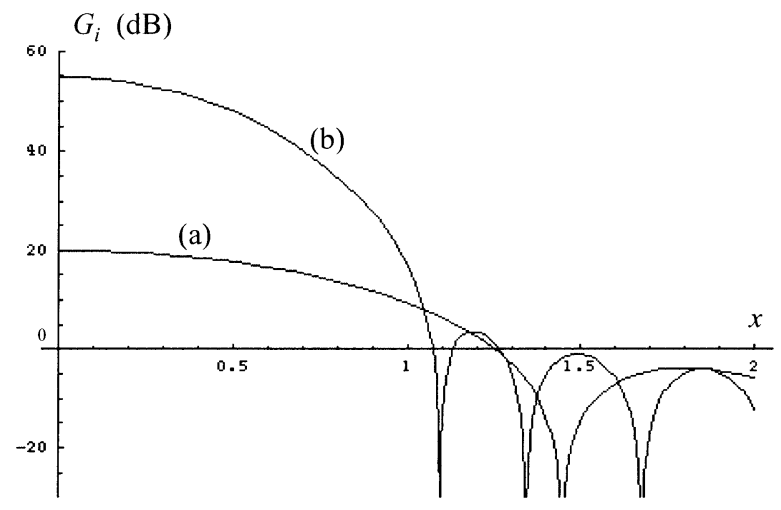

Fig. 2. Idler conversion efficiency $G_{i}$ versus $x$. (a) $\Phi=3$. (b) $\Phi=7$.

dispersion region), to see whether it could be suitable for filtering applications. Then the spectral width is relatively narrow, and the graph of $\Delta \beta$ versus $\omega_{s}$ is well approximated by a straight line in that region. All these spectra are similar in appearance, as the signal-to-idler power conversion gain $G_{i}$ can be written as

$$
G_{i}=\left|\frac{\sinh \left(\sqrt{1-x^{2}} \Phi\right)}{\sqrt{1-x^{2}}}\right|^{2}
$$

where $\Phi=\gamma P_{0} L$ is the pump self-phase modulation (SPM) shift. $x=1+\Delta \beta / 2 \gamma P_{0}$ is a dimensionless variable, normalized in such a way that $x=0$ where the gain is maximum; $x= \pm 1$ at what we defined as the edges of the narrow gain region. $x$ can be interpreted as the normalized signal frequency in the narrow gain region. In this manner, all these gain spectra correspond to the one-parameter set of curves defined by (4). $G_{i}$ is an even function of $x$. As $x$ increases from zero to one, $G_{i}$ decreases monotonically from $\sinh ^{2}(\Phi)$ to $\Phi^{2}$. As $x$ increases beyond one, the square root becomes imaginary, and $G_{i}$ behaves in a manner similar to a squared sinc function: the relative heights of the maxima are the same as for such a function, but the zeros are not evenly spaced along the $x$ axis because of the presence of $\sqrt{1-x^{2}}$ rather than $x$. Fig. 2 shows the graphs of $G_{i}$ for two values of $\Phi$. The central shape of the spectrum is not very flat. It is more like the so-called Gaussian shape of a squared sinc function; such shapes are satisfactory for a number of communication applications.

An important quantity is the ratio of the maximum gain to that of the first peak $\rho$, which determines the suitability for use as a narrow-band filter; it is approximately given by

$$
\rho=\left|\frac{3 \pi}{2} \frac{\sinh (\Phi)}{\Phi}\right|^{2} .
$$

This is a rapidly increasing function of $\Phi$. For $\Phi=3(7)$, which correspond to peak gains of 20(55) dB, we have $\rho=24(51) \mathrm{dB}$; i.e., we see that $\rho$ in decibels is of the same order as the peak gain in decibels. Since fiber OPAs with gains up to $65 \mathrm{~dB}$ have been demonstrated, we conclude that values of $\rho$ in the $20-60 \mathrm{~dB}$ range should in principle be realizable. (This of course assumes that a perfectly uniform fiber is used; longitudinal variations of 
$\lambda_{0}$ will alter this conclusion for very small bandwidths.) Sidelobe rejection ratios in this range are competitive with those of state-of-the-art passive filters.

We have assumed that the OPA is used as a wavelength converter in order to have simple expressions, as well as to obtain a high $\rho$. If the OPA is used as an amplifier, then the signal power gain $G_{s}=G_{i}+1$ applies, and $\rho$ is reduced. For large gains, however, the difference is negligible. Hence, such OPAs could be used as filters in a similar manner, either as amplifiers or as wavelength converters.

\section{Width of the Narrow Gain Spectrum}

We define the boundaries of the narrow gain region as $u_{1}$, where $\Delta \beta=0$, and $u_{2}$, where $\Delta \beta=-4 \gamma P_{0}$, Fig. 1 . We find that

$$
u_{1}=-\frac{12 \beta_{2}}{\beta_{4}} \quad \text { and } \quad u_{2}=-\frac{6 \beta_{2}}{\beta_{4}}\left(1+\sqrt{1-\frac{4 \gamma P_{0} \beta_{4}}{3\left(\beta_{2}\right)^{2}}}\right) .
$$

From $u_{1}$, we obtain the distance of the gain region from the pump in terms of frequency, $\Delta \omega_{1}=\left(u_{1}\right)^{1 / 2}=$ $\left(-\left(12 \beta_{2} / \beta_{4}\right)\right)^{1 / 2}$. Expressed in wavelength units, this becomes

$$
\Delta \lambda_{1} \approx \frac{\Delta \omega_{1}}{C}=\frac{1}{C}\left(-\frac{12 \beta_{2}}{\beta_{4}}\right)^{1 / 2}
$$

where $C=\left(2 \pi c / \lambda_{0}^{2}\right)$; for $\lambda_{0}=1550 \mathrm{~nm}, C=7.85 \times$ $10^{20} \mathrm{~s}^{-1} \mathrm{~m}^{-1}$. (The approximately equal sign is used in (6) to indicate that this conversion becomes less accurate as $\Delta \omega_{1} / \omega_{p}$ increases.)

If $\left|\left(4 \gamma P_{0} \beta_{4} / 3\left(\beta_{2}\right)^{2}\right)\right| \ll 1$ the gain bandwidth $\delta \omega=$ $\left|\Delta \omega_{1}-\Delta \omega_{2}\right|$ is relatively small compared to $\Delta \omega_{1}$, and it is well approximated by $\delta \omega=24\left|\left(\gamma P_{0} / \beta_{4}\left(\Delta \omega_{1}\right)^{3}\right)\right|$; in terms of wavelengths

$$
\delta \lambda \approx \frac{24}{C^{4}}\left|\frac{\gamma P_{0}}{\beta_{4}\left(\Delta \lambda_{1}\right)^{3}}\right|
$$

This form shows that the gain bandwidth decreases rapidly with distance from the pump: for example, the gain bandwidth $200 \mathrm{~nm}$ away from the pump will only be $1 / 8$ of the bandwidth $100 \mathrm{~nm}$ from the pump.

Another important consequence of (7) is that $\delta \omega$ scales linearly with $\gamma / \beta_{4}$. If we compare HNLF to DSF, HNLF has a $\gamma$ about ten times larger and a $\beta_{4}$ about ten times smaller than DSF. Hence, HNLF will yield a $\delta \omega$ which is about 100 times larger than DSF (provided that $P_{0}$ is the same, which can be done by using different fiber lengths to have the same gain). This is a very large ratio, which indicates that HNLF and DSF will yield rather different-looking spectra, which are likely to find different applications.

Fig. 3 shows the graph of $\delta \lambda$ versus $\Delta \lambda_{1}$ for DSF. If we want $\Delta \lambda_{1}=100(200) \mathrm{nm}$, we have $\delta \lambda=3.5(0.44) \mathrm{nm}$. These values of $\delta \lambda$ are desirable for optical communication systems, as they could accommodate one multigigahertz communication channel. For HNLF, the corresponding values of $\delta \lambda$ would be much larger, and might be useful for other applications, such as

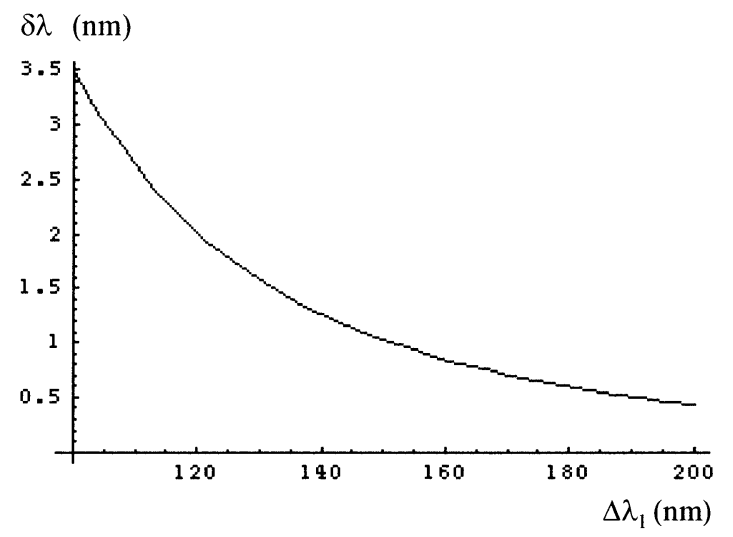

Fig. 3. Graph of gain bandwidth $\delta \lambda$ versus $\Delta \lambda_{1}$ for DSF.

amplification in spectral regions where there are no good optical amplifiers.

\section{Pump Wavelength Shift Required for Shifting the Gain Region}

Let us now determine the shift in pump wavelength $\Delta \lambda_{p}=$ $\lambda_{p}-\lambda_{0}$ required to have the gain region at $\Delta \lambda_{1}$ from the pump. It can be shown that

$$
\Delta \lambda_{p}=\frac{C}{12} \frac{\beta_{4}}{\beta_{3}}\left(\Delta \lambda_{1}\right)^{2} .
$$

For DSF $\Delta \lambda_{p}=-2.87 \times 10^{-4}\left(\Delta \lambda_{1}\right)^{2}$, where the wavelengths are in nanometers. For example, if $\Delta \lambda_{1}=100(200)$ $\mathrm{nm}, \Delta \lambda_{p}=-2.87(-11.5) \mathrm{nm}$. Hence, we see that the gain region can be shifted by several hundred nanometers by shifting the pump by just a few nanometers. This is convenient in practice, since pumps that can be tuned over tens of nanometers can readily be obtained in several spectral regions, particularly in the $C$ - and $L$-band. Redoing the calculations for HNLF, one finds that $\Delta \lambda_{p}=-0.77 \times 10^{-4}\left(\Delta \lambda_{1}\right)^{2}$ nm, i.e., 3.6 times smaller than for DSF.

\section{E. Pump Stability Requirements}

On the negative side, the large sensitivity of $\Delta \lambda_{1}$ to changes in $\lambda_{p}$ indicates that when the gain region is very narrow even a slight change in $\lambda_{p}$ will potentially shift the gain region by a fraction of its width, which would not be acceptable in applications such as narrow-band filtering. To quantify this, we calculate the excursion in pump wavelength $\delta$, which leads to a shift of the gain region equal to its width $\delta \lambda$. It is given by

$$
\delta=\frac{4}{C^{3}} \frac{\gamma P_{0}}{\left|\beta_{3}\right|\left(\Delta \lambda_{1}\right)^{2}}
$$

In order for the gain region to be essentially unaffected by possible drift in pump wavelength, such drift should be small compared to $\delta$. For $\Delta \lambda_{1}=100(200) \mathrm{nm}$, we find that for DSF $\delta=0.2(0.05) \mathrm{nm}$, and for HNLF $\delta=6.1(1.52) \mathrm{nm}$. For DSF these numbers correspond to a few gigahertz, which indicates that a stable pump wavelength will be necessary to accurately maintain the position of the gain region. For HNLF pump wavelength stability is far less critical. 
The pump intensity should also be stable. If the pump has significant relative intensity noise (RIN), it will be transferred to the signal and the idler, with a magnification factor which is of the order of $2 \phi$ at maximum gain [7].

\section{F. Effect of Longitudinal Variations of $\lambda_{0}$}

Practical fibers exhibit longitudinal variations of $\lambda_{0}$, with a distribution including both long-range and short-range components. Short-range variations are not a problem, as they can be handled by means of their local average value. For long-range variations, however, averaging is not appropriate, and the actual spatial map of $\lambda_{0}$ must be accurately taken into account. The effect on four-wave mixing of such $\lambda_{0}$ variations has been investigated in detail [8].

Since OPA performance depends on $\Delta \lambda_{p}=\lambda_{p}-\lambda_{0}$, we can then reinterpret the preceding discussion about pump wavelength fluctuations in terms of the effect of longitudinal variations of $\lambda_{0}$. These will cause variations of $\beta_{2}$ and, hence, of $\Delta \lambda_{1}$. Thus, if we consider a realistic fiber, the location of the gain region will change along the fiber. As a result, the input-tooutput gain spectrum, which we may approximatively think of as the integral of the local gain spectra along the fiber, will be broadened, and the peak gain will be reduced. Since $\lambda_{0}$ and $\lambda_{p}$ play similar roles in $\Delta \lambda_{p}$, we see that $\delta$ can also be interpreted as the variation in $\lambda_{0}$ necessary to displace the gain curve by an amount equal to its width. Hence, we can say that $\delta$ is the amount of variation of $\lambda_{0}$ along the fiber that will lead to a doubling of the gain bandwidth. The numerical values calculated above for DSF indicate that $\lambda_{0}$ should not vary by more than a few hundredths of a nanometer along the fiber if we want to maintain narrow high-gain regions far from $\lambda_{0}$. Since it is known that typical DSF can exhibit $\lambda_{0}$ variations of the order of one nanometer over lengths of several kilometers [9], [10], the fiber should be short in order to be able to observe such gain regions. This is why in the experiments we used $L=200 \mathrm{~m}$ for DSF.

We can also use this result as a qualitative measurement of the spread of $\lambda_{0}$ over the fiber length: if in some experimental conditions we observe a doubling of the gain bandwidth (compared to its theoretical value), then the spread in $\lambda_{0}$ over that length is of the order of $\delta$.

\section{G. Effect of Temperature Variations}

It is well known that $\lambda_{0}$ is a function of temperature. In DSF $\lambda_{0}$ varies at the rate of about $0.03 \mathrm{~nm} /{ }^{\circ} \mathrm{C}$ [11]; in HNLF the rate is $0.06 \mathrm{~nm} /{ }^{\circ} \mathrm{C}$ [12]. Thus, for a $1{ }^{\circ} \mathrm{C}$ change in DSF, we have a $0.03-\mathrm{nm}$ shift, which is comparable to $\delta$ calculated in Section II-C for $\Delta \lambda_{1}=200 \mathrm{~nm}$. This shows that such a small temperature change, if uniform over the fiber, would shift the gain region by a whole width, thereby potentially affecting its operation as a narrow filter. Also, if the temperature changes are nonuniform along the fiber, they will introduce a longitudinal variation of $\lambda_{0}$, with the same detrimental effects as in the preceding section. These considerations indicate that careful temperature control would be required for accurate operation of such filters.
On the positive side, one may envision designs wherein the temperature of a number of fiber sections can be independently controlled. This would then in principle allow one to obtain a variety of spectral shapes. In particular, one might be able to implement a three- or four-section scheme proposed for flattening the broad gain spectrum of one-pump fiber OPAs [13].

To quantify this effect, let us define $K_{\mathrm{th}}$ as the rate of change of $\lambda_{0}$ with temperature $T$. It can then be shown that the rate of shift of the gain region with temperature is

$$
\frac{d\left(\Delta \lambda_{1}\right)}{d T}=\frac{6 K_{\mathrm{th}} \beta_{3}}{C \beta_{4} \Delta \lambda_{1}} .
$$

The $1 / \Delta \lambda_{1}$ dependence shows that the extreme spectra shift less than the ones close to $\lambda_{0}$. However, since the width $\delta \lambda$ of the peaks is proportional to $1 /\left(\Delta \lambda_{1}\right)^{3}$, we see that the extreme spectra experience temperature-induced shifts which are much larger relative to their widths than the ones close to $\lambda_{0}$. This could cause greater difficulties for the extreme spectra in filtering-type applications.

\section{H. Effect of Fiber Birefringence}

Fibers such as DSF and HNLF exhibit nearly linear birefringence. Its magnitude and the orientation of its principal axes vary randomly along their length; they may also vary in time due to temperature changes, acoustic vibrations, etc. The beat length is of the order of $1 \mathrm{~m}$. Since the gain of fiber OPAs depends on the states of polarization (SOPs) of the waves [14], one must actually consider that at each point along the fiber the local gain spectrum has a slightly different shape. As a result, the end-to-end OPA gain spectrum can be thought of as being the spatial average of the local gains. Clearly this means that this gain spectrum will not be quite the same as in a fiber in which all SOPs remain linear and parallel over the entire length, as considered so far in the gain calculations.

The theory of fiber OPAs with waves in a variety of SOPs shows that the range of $\Delta \beta$ over which gain is present is reduced in width and shifted by an amount of the order of $\gamma P_{0}$; also the maximum gain is reduced. Looking at Fig. 1, we see that for large $\Delta \lambda_{1}$ these kinds of changes will lead to shifts in the location of the gain spectrum that are of the order of $\delta \lambda$. Hence, we can anticipate that random birefringence could lead to spectrum broadening, but at most by a factor of two.

In long fibers, longitudinal variation of $\lambda_{0}$ can lead to spectral broadening by many $\delta \lambda$ 's; hence, birefringence broadening should play a negligible role in such fibers. On the other hand, in very short fibers with very little $\lambda_{0}$ variation, birefringence broadening will dominate.

Fibers that are tens or hundreds of meters long, such as used in our experiments, are many beat lengths long. Therefore, SOPs will generally evolve considerably along their lengths, and the end-to-end gain spectra will necessarily differ somewhat from the ideal ones shown in Fig. 2. Accurate observation of the ideal spectra would require the use of special low-birefringence fibers, such as twisted fibers [15] or spun fibers [16]. 


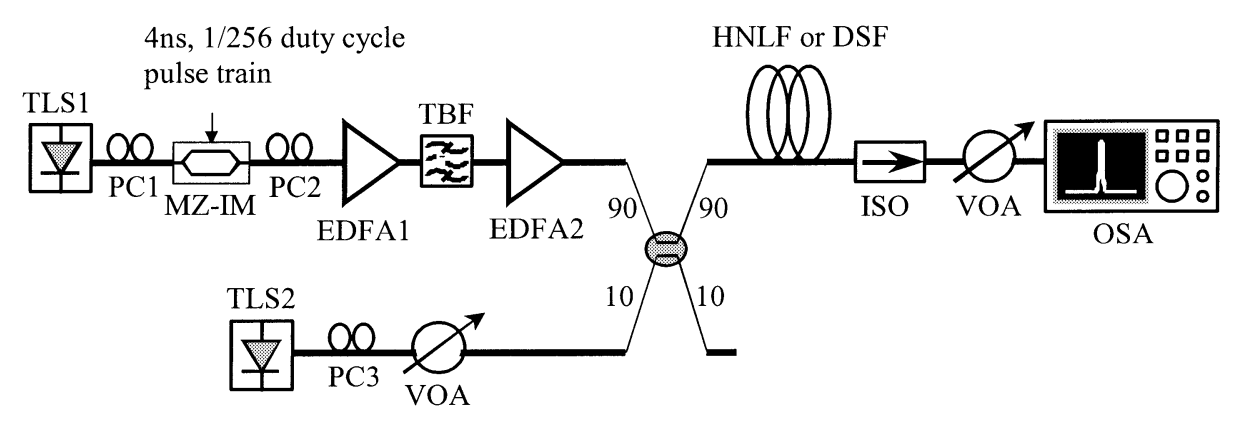

Fig. 4. Experimental setup.

\section{EXPERIMENTS}

\section{A. Measurement Procedure}

Ideally the gain of an optical amplifier should be measured by feeding into it a low-power signal at the desired wavelength, and measuring the resulting output signal. However, for experiments such as ours, where the wavelength range of interest is about $1300-1800 \mathrm{~nm}$, it is very difficult to find suitable signal sources. For this reason, we resorted to the measurement of amplified spontaneous emission (ASE) as a substitute for gain measurements. This is possible because in a lossless OPA with low noise figure (i.e., NF near $3 \mathrm{~dB}$ ), there is a linear relationship between ASE output power and gain, since the output ASE can be viewed as vacuum fluctuations at the input amplified by a noiseless amplifier [17]. Of course, since in practice we do not know exactly the OPA NF, this only provides an approximate gain measurement. However, for our main purpose here of measuring gain bandwidths, this relationship is adequate.

Caution is necessary to apply this method to fiber OPAs. The main difficulty is that at high gain ASE power density in the fiber can become very high. This can lead to two problems.

1) The first one is that saturation can be reached, leading to pump depletion, and an output ASE spectrum which is no longer a close replica of the small-signal gain spectrum.

2) Another problem is that because the OPA is operated near $\lambda_{0}$, fiber dispersion is low. As a result most types of fourwave mixing (FWM) interactions are well phase matched, and spurious FWM products can become sizable if some of the interacting waves are large. This can often be seen in spectra exhibiting a narrow peak, which acts as a secondary pump. Then the ASE spectrum around such a peak tends to become symmetric, even if low-power theory predicts a strong asymmetry: this is a clear sign of FWM among ASE components near that peak.

In practice, then, one has to find an optimum: if gain is too low, the ASE level may be too low to be detected; if gain and ASE level are too high, the above problems may strongly distort the ASE spectra. To find this optimum, it is very useful to have a good simulation of the gain spectra, so that one can judge when the resemblance with the experimental data is greatest, indicating a tolerable level of spurious nonlinear effects. The optimization is different for different fibers, and this explains why we used different powers for our measurements in DSF and HNLF.

We have also occasionally measured the actual gain spectra, over limited spectral ranges where sources were available. We did this both to validate and calibrate the ASE-based procedure, and to measure record gains. The gain was the on-off gain, measured by taking the ratio of the output signal power with the pump on to that with the pump off.

\section{B. Experimental Setup}

The experimental configuration is shown in Fig. 4. The gain medium is either DSF or HNLF, with the parameters listed in Section II-A. In both cases, the pump source is a tunable laser source, TLS1, which is tuned in order to obtain a whole family of gain spectra. It is pulse-modulated with a 4-ns pulsewidth and $1 / 256$ duty cycle, to provide $12 \mathrm{~W}$ of peak power in DSF, or $20 \mathrm{~W}$ in HNLF (after amplification). Polarization controller PC1 aligns the pump's SOP with the transmission axis of the Mach-Zehnder intensity modulator (MZ-IM). The pump is then amplified by EDFA1, and filtered by a 0.35 -nm bandwidth tunable bandpass filter (TBF). It is further amplified by EDFA2, with a maximum average output power of $27 \mathrm{dBm}$.

Due to the limited power budget, we cannot insert another TBF after EDFA2 as we did in [3]; as a result, the ASE of EDFA2 is injected into the OPA However, no significant gain saturation attributable to EDFA ASE is observed. The OPA ASE spectrum is clearly seen under most circumstances. An exception is in the 1520-1580-nm range (which includes the pump), where significant EDFA ASE is amplified by the OPA, and cannot always be distinguished from OPA ASE. This, however, is of little consequence here, as we are most interested in OPA gain spectra with maxima outside of this central region.

Two other TLSs, tunable in the 1400-1550-nm and 1500-1650-nm ranges, were also used as signal sources to measure the OPA gain in order to relate the OPA gain and ASE spectra in certain regions. Maximum OPA gain and conversion efficiency is achieved by aligning the SOPs of signal and pump by PC3. Signal and pump are combined by a 90/10 coupler, and then enter the OPA fiber. The signal input power to the OPA is adjusted by a variable optical attenuator (VOA). After another isolator, which prevents any reflection from the VOA, the output spectrum of the OPA is observed at the optical spectrum analyzer (OSA).

\section{RESULTS}

\section{A. $H N L F$}

We first performed experiments with HNLF, because the predicted bandwidths are much larger than for DSF. Hence, we expected that we should be able to obtain fairly wide gain regions far away from the pump, that might for example be useful for 


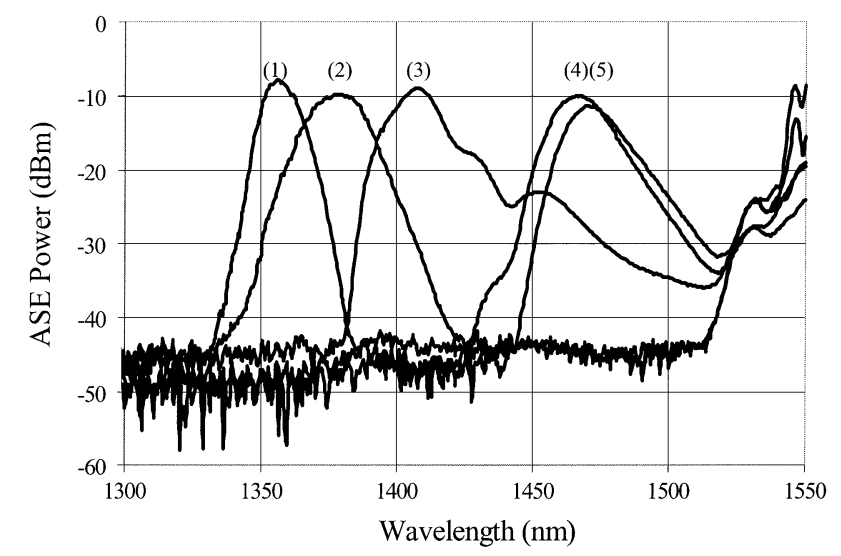

Fig. 5. Experimental ASE spectra for HNLF. Temperature $=25^{\circ} \mathrm{C}$. The $\lambda_{p}$ 's in nanometers are: (1) 1553 ; (2) 1553.8 ; (3) 1555.4 ; (4) 1557 ; and (5) 1557.8 . The average $\lambda_{0}$ was $1556 \mathrm{~nm}$.

amplification near $1310 \mathrm{~nm}$. We settled on a peak pump power of about $20 \mathrm{~W}$ for small value of $\Delta \lambda_{p}$, as it gave reasonable ASE spectral shapes.

Decreasing $\lambda_{p}$, we found that initially the maximum ASE values remained constant, in agreement with the fact that the maximum gain given by (1) only depends on $\Phi=\gamma P_{0} L$, and is independent of the particular shape of the gain spectrum. This held until $\lambda_{p} \approx \lambda_{0}$, which corresponds to the widest one-piece gain spectrum. When $\lambda_{p}$ was decreased below $\lambda_{0}$ (normal dispersion), the spectral shape changed as predicted by theory: it consisted of a central peak around the pump, and two isolated peaks far from the pump. However, we found that the ASE peak dropped more and more. As discussed in Section II-F, this is an indication that $\lambda_{0}$ varies along the fiber. To record the data, we increased the pump power to maintain the same peak for all spectra; we stopped recording when we reached the highest pump power that we could obtain. The advantage of recording the data this way is that it then looks quite similar to simulations for fibers with uniform $\lambda_{0}$. Of course, to quantitatively interpret the data, one should remember that $P_{0}$ is higher for the extreme spectra.

The ASE data is shown in Fig. 5. Since OPA gain spectra are symmetric with respect to the pump when plotted with respect to $\Delta \omega_{s}$, we have shown the spectra only on one side of the pump. Because the range of the OSA ends at $1700 \mathrm{~nm}$, the long-wavelength parts of the more interesting gain spectra were not available; for this reason, we show only the short-wavelength parts of the spectra, which were fully displayed by the OSA. Fig. 6 is a simulation of the gain spectra, using the experimental parameters. We note that there is fair agreement between the shapes of the experimental ASE and simulated gain spectra.

Since plot (1) is the most extreme gain spectrum that we were able to obtain, we fell short in our attempt to make an amplifier operating near $1310 \mathrm{~nm}$, with a pump in the $C$-band. As discussed above, the limiting factor is the variation of $\lambda_{0}$ along the fiber.

An important item is that plot (3) of Fig. 5, corresponding to $\lambda_{p} \approx \lambda_{0}$, is to the best of our knowledge the widest onepiece ASE spectrum observed to date for any fiber OPA. The spectrum rises abruptly at about $1380 \mathrm{~nm}$, and we take this to correspond to the lower limit of the gain spectrum. The pump is at $1555.4 \mathrm{~nm}$. The upper limit of the gain spectrum is calculated

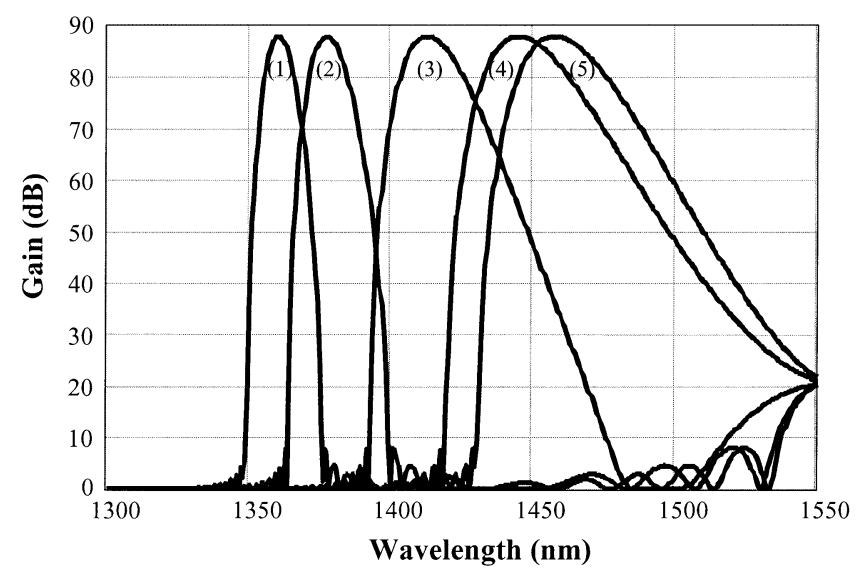

Fig. 6. Theoretical gain spectra for HNLF. Temperature $=25^{\circ} \mathrm{C}$. The $\lambda_{p}$ 's are as in Fig. 5.

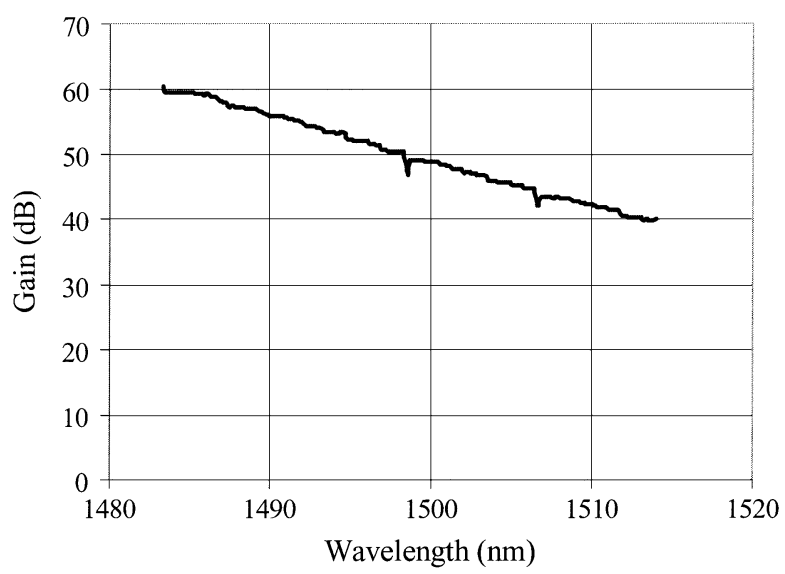

Fig. 7. Experimental gain spectrum for HNLF. $\lambda_{p}=1557.8 \mathrm{~nm}$.

by using the preceding symmetry property, and is found to be $1782 \mathrm{~nm}$. Hence, we infer a 400-nm gain bandwidth, slightly larger than the $360 \mathrm{~nm}$ that we recently obtained with the same fiber [3]; the difference can be attributed to the higher pump power used here.

Concerning the widths of (1) and (2), we see that the experimental values are wider than the theoretical ones by about a factor of two. According to our discussion of Section II-F, this can be attributed to the presence of $\lambda_{0}$ variations. This is also consistent with the fact that for these graphs $P_{0}$ had to be raised to maintain the peak value. According to our reasoning in Section II-F, we can obtain an estimate of the extent of the $\lambda_{0}$ variation over the fiber length by finding where the experimental width of the narrow gain peak is about twice the theoretical value. We note that this occurs for curve (1), for which $\Delta \lambda_{1} \approx 200 \mathrm{~nm}$. From the calculation in Section II-E, we have $\delta=1.52 \mathrm{~nm}$, which is the estimate for the $\lambda_{0}$ variation. This variation takes place along a fiber which is just $30 \mathrm{~m}$ long, and this leads to a rate of change with distance $\delta / L=50 \mathrm{~nm} / \mathrm{km}$. This is consistent with other measurements on a longer fiber of this type, that we have performed by cutting it into $100-\mathrm{m}$ pieces, and measuring the average $\lambda_{0}$ of each piece by maximizing OPA gain bandwidth.

We have also measured the OPA gain directly by amplifying an input signal. Fig. 7 shows the gain spectrum obtained for $1557.8 \mathrm{~nm}$. We see that the linear shape of the gain curve 


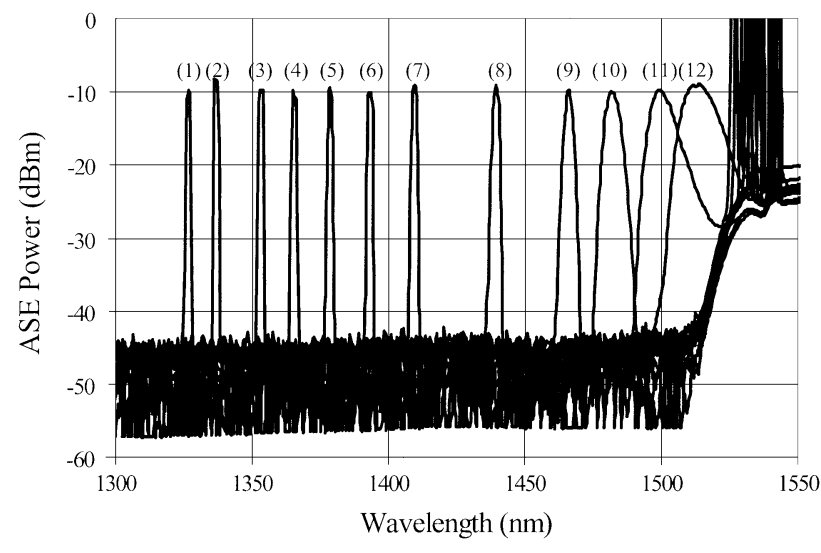

Fig. 8. Experimental ASE spectra for DSF. Temperature $=25^{\circ} \mathrm{C}$. The $\lambda_{p}$ 's in nanometers are: (1) 1526.6 ; (2) 1528.1 ; (3) 1530.4 ; (4) 1532 ; (5) 1533.5 ; (6) 1535.1 ; (7) 1536.6 ; (8) 1538.9; (9) 1540.5; (10) 1541.3; (11) 1542; and (12) 1542.8. The average $\lambda_{0}$ was $1539.3 \mathrm{~nm}$.

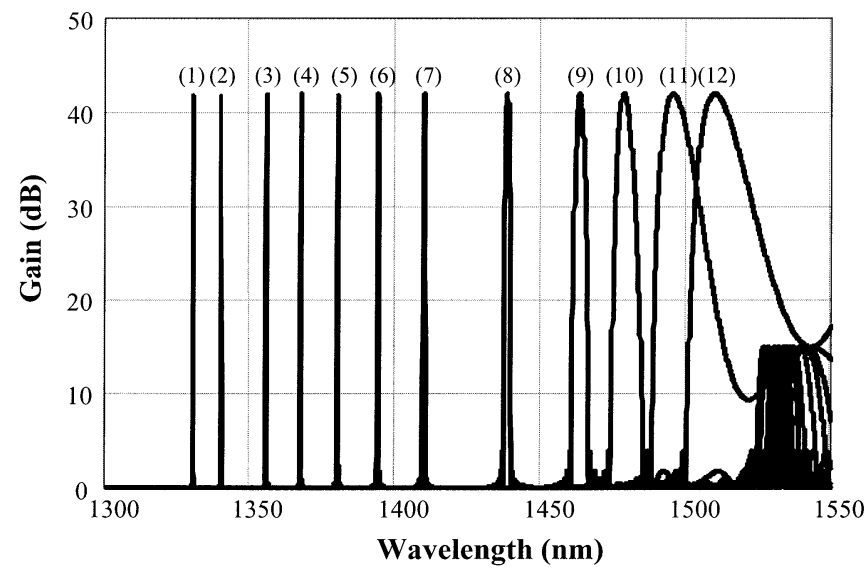

Fig. 9. Theoretical gain spectra for DSF. Temperature $=25^{\circ} \mathrm{C}$. The $\lambda_{p}$ 's are as in Fig. 7.

matches well that of the corresponding ASE curve in Fig. 5. In a separate measurement, we obtained an on-off gain of $65 \mathrm{~dB}$ for $\lambda_{s}=1482 \mathrm{~nm}$. To our knowledge, this is the highest on-off gain reported for any fiber OPA.

\section{B. $D S F$}

We then performed experiments with DSF, which is known to have much smaller $\lambda_{0}$ variations than HNLF and might, thus, allows us to obtain narrow gain regions further from the pump than HNLF.

We first performed ASE measurements as for HNLF, at room temperature. The results are shown in Fig. 8. Fig. 9 is a simulation of the OPA gain spectra, with the experimental parameters. We see that there is good agreement between experimental and simulated results. (This provides an experimental verification of the proportionality between OPA ASE and gain that we justified on theoretical grounds in Section III-A.) As for HNLF, we found it necessary to increase $P_{0}$ to maintain the peak ASE value for the more extreme spectra.

As anticipated in Section II, the gain regions for DSF are much narrower than for HNLF. However, since the $\lambda_{0}$ variations in DSF are very small, we can actually go further than with HNLF before ASE amplitude cannot be maintained. We were able to reach about $1325 \mathrm{~nm}$, but still fell short of $1310 \mathrm{~nm}$. (a)

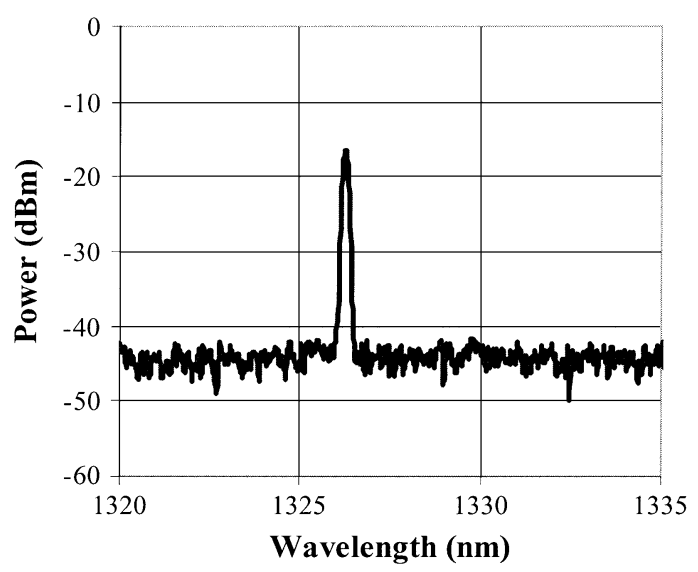

(b)

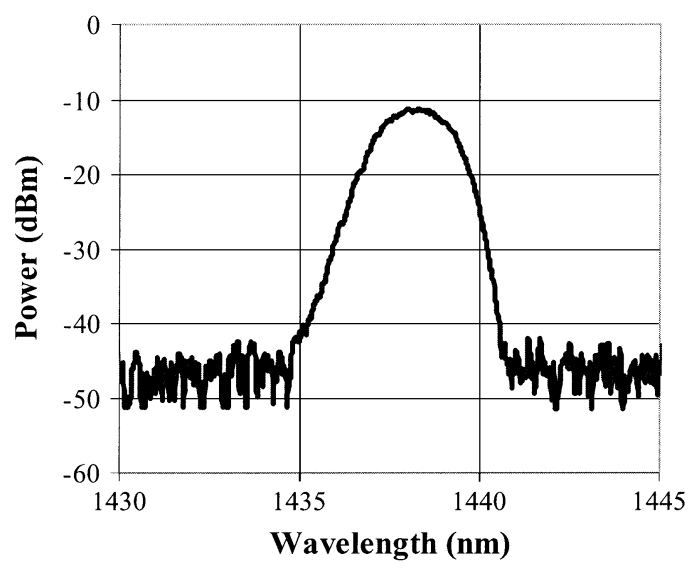

Fig. 10. Expanded views of narrow gain spectra of Fig. 8. (a) $\lambda_{p}=$ $1526.6 \mathrm{~nm}$. (b) $\lambda_{p}=1538.9 \mathrm{~nm}$.

Since the width and shape of the extreme gain spectra are difficult to see on Fig. 8, we have magnified two of them in Fig. 10. Fig. 10(a) shows the most extreme spectrum. It has a width which is below $1 \mathrm{~nm}$, approaching the resolution of the OSA $(0.1 \mathrm{~nm}$ here); for that reason, its shape is not well resolved. Fig. 10(b) shows an intermediate spectrum, with a larger width; its shape is well resolved by the OSA. Its width is about $6 \mathrm{~nm}$. The width for the corresponding simulated gain spectrum in an ideal fiber is about $2.5 \mathrm{~nm}$. Thus, we see that we have approximately a doubling of the bandwidth, attributable to $\lambda_{0}$ variations. Since $\Delta \lambda_{1} \approx 100 \mathrm{~nm}$, the example of Section II-E yields $\delta=0.05 \mathrm{~nm}$, which we take to be an estimate of the $\lambda_{0}$ variations in DSF. Since $L=200 \mathrm{~m}$, we obtain an estimate for the rate of change of $\lambda_{0}, \delta / L=0.25 \mathrm{~nm} / \mathrm{km}$. This is more than two orders of magnitude smaller than for HNLF. This explains why we were able to observe high-amplitude ASE spectra further from $\lambda_{0}$ with DSF than with HNLF: even though the spectra are much narrower for DSF, the high $\lambda_{0}$ uniformity of DSF preserves the near-theoretical shape of the gain spectra further away from $\lambda_{0}$ for DSF than for HNLF.

It is worth mentioning that the spectrum of Fig. 10(b), though about twice as wide as the ideal one, still has a shape which is fairly similar to that of Fig. 2, except for a slight asymmetry. While such a spectrum shape might not be suitable for filtering in communication systems, it could be adequate for an ASE light source. 
We showed in Section II-G that because the gain spectra in DSF are very narrow, even a slight shift in $\lambda_{0}$ due to a temperature change can shift them by several times their width, which could have a detrimental effect in a number of applications. We have performed an experiment to observe this effect in DSF. Since we already had data at room temperature $\left(25^{\circ} \mathrm{C}\right)$, we took similar data at $0{ }^{\circ} \mathrm{C}$, by placing the fiber spool in a mixture of water and ice. We found that peak (8) of Fig. 8 was shifted by about $10 \mathrm{~nm}$. In agreement with Section II-G, the extreme spectra were found to shift less than the ones close to $\lambda_{0}$, but that their shifts were much larger relative to their widths.

\section{Discussion}

The agreement between the experimentally measured ASE spectra shown and the theoretically calculated gain spectra is good. The experimental ASE spectra did not include wavelengths longer than $\lambda_{p}$ and, thus, did not show possible spontaneous Raman scattering. We did notice ASE peaks attributable to SRS in our data, about $100 \mathrm{~nm}$ on the long-wavelength side of the pumps, but their amplitudes were always considerably smaller than those of the peaks attributable to parametric gain only. This is in agreement with previous measurements of gain in broadband amplifiers combining parametric and Raman gain [2], where we found that the Raman gain could be considered to be only a small perturbation compared to the parametric gain.

An interesting aspect of obtaining a fairly wide gain region located far from the pump is that if that region is used to simultaneously amplify several carriers in a wavelength-division multiplexing (WDM) system, FWM between these carriers will be reduced, compared to an amplifier operating near $\lambda_{0}$. The reason for this is that the fiber chromatic dispersion coefficient $D$ in that region will be larger than near $\lambda_{0}$. For example, for HNLF with a dispersion slope $D_{\lambda} \approx 0.03 \mathrm{ps} \mathrm{nm}{ }^{-2} \mathrm{~km}^{-1}$, a gain region $200 \mathrm{~m}$ from $\lambda_{0}$ will have $D \approx 6 \mathrm{ps} \mathrm{nm}^{-1} \mathrm{~km}^{-1}$. This fairly high dispersion value should help reduce the spurious FWM terms, which are a cause for concern in fiber OPAs operating near $\lambda_{0}$ [18], [19].

This work indicates that the rate of change of $\lambda_{0}$ in HNLF is about two orders of magnitude larger than in DSF. The large $\lambda_{0}$ variations in HNLF rapidly broaden the narrow gain regions as they move away from $\lambda_{0}$, making it impossible to push them by more than $200 \mathrm{~nm}$. Our understanding is that HNLFs are currently manufactured by a drawing process which is different from the one used for DSF and that that is mostly what causes the large $\lambda_{0}$ variations. If HNLFs could be fabricated by a process similar to that for DSF, and, thus, exhibit $\lambda_{0}$ variations similar to those of DSF, it would then be possible to push these gain regions much further, possibly below $1200 \mathrm{~nm}$ (and above $2100 \mathrm{~nm}$ for the Stokes). This could, for example, be used for making a tunable ASE source operating over nearly an octave.

We have attributed the drop in ASE intensity as $\Delta \lambda_{1}$ increases primarily to $\lambda_{0}$ variations. Other phenomena may also contribute to the drop. They are related to the fact that singlemode fibers are typically not designed to operate over wavelength ranges as large as we are interested in in this work. On the short-wavelength side, single-mode fibers have a cutoff wavelength, below which a few modes can propagate. On the long wavelength side, the mode confinement decreases, and this leads to an increase in bend losses. A better understanding of the impact of these effects on fiber OPAs will be needed to optimize the design of OPAs operating over even wider ranges.

We have suggested that the narrow gain regions could potentially be used as narrow-band filters with gain. However, since high gain is required to obtain high selectivity, there is a possibility that strong input signals could drive the OPA into saturation. This is of course a possible disadvantage compared to passive filters, which are not subject to this limitation.

During the reviewing process a reviewer pointed out the existence of [20]. The fundamental physical mechanism responsible for the observations of [20] is the same as in our paper, namely, the fact that in a fiber with $\beta_{4}<0$, placing the pump in the normal dispersion region creates isolated gain regions away from the pump. In [20], the measurements were performed by means of modulation instability (MI), whereas we used OPA ASE, as well direct parametric gain measurement on an input signal. Hence, we obtained complementary information on the same basic physical phenomenon. The existence of these parallel efforts illustrates once again the close connection between MI and parametric amplification in fibers and the fact that researchers in one area can often benefit from research results in the other area.

\section{CONCLUSION}

By tuning the pump wavelength in the normal dispersion region near the zero-dispersion wavelength in a one-pump fiber OPA, we have observed, for the first time to our knowledge, the wide range of gain spectra theoretically obtainable from such OPAs. With highly nonlinear fiber, we have obtained the widest one-piece gain spectrum reported to date, with an inferred bandwidth of $400 \mathrm{~nm}$; we have also obtained $65 \mathrm{~dB}$ of on-off gain, the highest value reported for any fiber OPA. We have also observed gain regions tens of nanometers wide, as far as $200 \mathrm{~nm}$ from the pump. Such gain regions could provide gain in spectral regions where good amplifiers currently do not exist. With common dispersion-shifted fiber, and a pump near $1530 \mathrm{~nm}$, we have observed gain regions as narrow as $1 \mathrm{~nm}$, up to $200 \mathrm{~nm}$ from the pump. Such gain regions could be used for tunable filtering with gain. Alternatively, they could be used as tunable sources of narrow-band ASE. We have also measured the temperature dependence of these gain spectra, which could affect the performance of narrow-band devices, but also offers possibilities for spectral shaping. By documenting the rich variety of gain spectra obtainable from one-pump fiber OPAs, this work opens the way for the possible application of fiber OPAs to novel areas such as narrow-band filtering, narrow-band ASE generation, or amplification in regions far from the pump.

\section{REFERENCES}

[1] M. E. Marhic, N. Kagi, T.-K. Chiang, and L. G. Kazovsky, "Broadband fiber optical parametric amplifiers," Opt. Lett., vol. 21, pp. 573-575, 1996.

[2] M.-C. Ho, M. E. Marhic, Y. Akasaka, and L. G. Kazovsky, "200-nm bandwidth fiber optical amplifier combining parametric and Raman gain," J. Lightwave Technol., vol. 19, pp. 977-981, July 2001.

[3] K. K. Y. Wong, M. E. Marhic, G. Kalogerakis, and L. G. Kazovsky, "Fiber optical parametric amplifier and wavelength converter with record $360 \mathrm{~nm}$ gain bandwidth and $50 \mathrm{~dB}$ signal gain," presented at the Conf. Lasers and Electro-Optics 2003, Baltimore, MD, Postdeadline paper CThPDB6. 
[4] M. E. Marhic, Y. Park, T.-K. Chiang, and L. G. Kazovsky, "Tunable narrow bandpass filters with gain based on fiber optical parametric amplification," presented at the Conf. Lasers and Electro-Optics/Quantum Electronics and Laser Science Conf. 1996, Anaheim, CA, Paper QMB5.

[5] C. Lin, W. A. Reed, A. D. Pearson, and H.-T. Shang, "Phase matching in the minimum-chromatic-dispersion region of single-mode fibers for stimulated four-photon mixing," Opt. Lett., vol. 6, pp. 493-495, 1981.

[6] J. P. Pocholle, J. Raffy, M. Papuchon, and E. Desurvire, "Raman and four photon mixing amplification in single mode fibers," Opt. Eng., vol. 24, pp. 600-608, 1985.

[7] G. Kalogerakis, M. E. Marhic, K. K.-Y. Wong, and L. G. Kazovsky, "Pump to signal RIN transfer in fiber OPAs," presented at the Conf. Lasers and Electro-Optics 2004, San Francisco, CA.

[8] M. Karlsson, "Four-wave mixing in fibers with randomly-varying zerodispersion wavelength," J. Opt. Soc. Amer. B, vol. 15, pp. 2269-2275, 1998

[9] I. Brenner, P. P. Mitra, D. D. Lee, and D. J. Thomson, "High-resolution zero-dispersion wavelength mapping in single-mode fiber," Opt. Lett., vol. 23, pp. 1520-1522, 1998.

[10] J. M. Chavez-Boggio, S. Tenenbaum, and H. L. Fragnito, "Four wave mixing induced changes in the noise spectrum in an optical fiber," in Tech. Dig. Optical Fiber Communication Conf., 2001, pp. WDD24.1-WDD24-4.

[11] K. C. Byron, M. A. Bedgood, A. Finney, C. McGauran, S. Savory, and I. Watson, "Shifts in zero dispersion wavelength due to pressure, temperature, and strain in dispersion shifted singlemode fibers," IEE Electron. Lett., vol. 28, pp. 1712-1714, 1992.

[12] J. Hansryd, F. Dross, M. Westlund, P. A. Andrekson, and S. N. Knudsen, "Increase of the SBS threshold in a short highly nonlinear fiber by applying a temperature distribution," J. Lightwave Technol., vol. 19, pp. 1691-1697, Nov. 2001

[13] L. Provino, A. Mussot, E. Lantz, T. Sylvestre, and H. Maillotte, "Broadband and flat parametric amplifiers with a multisection dispersion-tailored nonlinear fiber arrangement," J. Opt. Soc. Amer. B, vol. 20, pp. 1532-1537, 2003

[14] M. E. Marhic, K. K.-Y. Wong, and L. G. Kazovsky, "Fiber optical parametric amplifiers with linearly- or circularly-polarized waves," J. Opt. Soc. Amer. B, vol. 20, pp. 2425-2433, 2003.

[15] Y. Liang, J. W. Lou, J. K. Andersen, J. C. Stocker, O. Boyraz, and M. N. Islam, "Polarization-insensitive nonlinear optical loop mirror demultiplexer with twisted fibers," Opt. Lett., vol. 24, pp. 726-728, 1999.

[16] F. Gutty, S. Pitois, P. Grelu, G. Millot, M. D. Thomson, and J. M. Dudley, "Generation and characterization of $0.6-\mathrm{THz}$ polarization domain-wall trains in an ultralow-birefringence spun fiber," Opt. Lett., vol. 24, pp. 1389-1391, 1999

[17] Y. Yamamoto and K. Inoue, "Noise in amplifiers," J. Lightwave Technol., vol. 21, pp. 2895-2915, Nov. 2003.

[18] T. Torounidis, H. Sumerud, P. O. Hedekvist, and P. A. Andrekson, "Amplification of WDM signals in fiber-based optical parametric amplifiers," IEEE Photon. Technol. Lett., vol. 15, pp. 1061-1063, Aug. 2003.

[19] F. A. Callegari, J. M. C. Boggio, and H. L. Fragnito, "Spurious four-wave mixing in two-pump fiber-optic amplifiers," IEEE Photon. Technol. Lett., vol. 16, pp. 434-436, Feb. 2004.

[20] S. Pitois and G. Millot, "Experimental observation of new modulation instability spectral window induced by fourth-order dispersion in a normally dispersive single-mode optical fiber," Opt. Comm., vol. 226, pp. 415-422, 2003.

Michel E. Marhic (M'79-SM'89) received the Diplome D'Ingenieur degree in electrical engineering from Ecole Superieure d'Electricite, the M.S. degree in electrical engineering from Case Western Reserve University, Cleveland, $\mathrm{OH}$, and the Ph.D. degree in electrical engineering from the University of California, Los Angeles.

He was on the faculty of the Department of Electrical Engineering, Northwestern University, Evanston, IL (1974-1998), and on sabbatical leaves at the University of Southern California, Los Angeles (1979-1980), and Stanford University, Stanford, CA (1984-1985 and 1993-1994). He is currently Consulting Professor in the Department of Electrical Engineering, Stanford University. $\mathrm{He}$ (co)-founded Holicon, Holographic Industries, and OPAL Laboratories. He is the author or coauthor of over 270 journal and conference papers and had eight patents awarded. Over the past 25 years, his research has been in several areas of applied optics, including nonlinear interactions in plasmas; optical fiber measurements; hollow infrared waveguides; holography and phase conjugation; and fiber networks. Over the past ten years, his emphasis has been on optical communication systems and on nonlinear optical interactions in fibers.

Dr. Marhic is a Member of the Optical Society of America and Eminent Member of Tau Beta Pi. He was recipient of the Ameritech Research Professorship from the Institute for Modern Communications (1990-1991).
Kenneth Kin-Yip Wong ( $\left.\mathrm{S}^{\prime} 00-\mathrm{M}^{\prime} 04\right)$ received the combined B.E. degree in electrical engineering and B.S. degree in physics from the University of Queensland, Brisbane, Australia, in 1997 and the M.S. and Ph.D. degrees in electrical engineering from Stanford University, Stanford, CA, in 1998 and 2003, respectively.

He was a Member of the Photonics and Networking Research Laboratory, Stanford University. His research field included DWDM systems, subcarrier multiplexing (SCM) optical systems, fiber nonlinearity, and fiber optical parametric amplifiers. He was with Hewlett-Packard Laboratories in 1998-1999 as Research Engineer and contributed to projects including parallel optics and vertical-cavity surface-emitting lasers (VCSELs). He was also an Independent Consultant for Innovation CORE (A Sumitomo Electric Company) in 2004. He is currently an Assistant Professor in the Department of Electrical and Electronic Engineering, University of Hong Kong, Hong Kong, China. He is author or coauthor of over 50 journal and conference papers. He is a Reviewer for Optics Letters, the Journal of the Optical Society of America B, and Optics Communications. His research interests include DWDM systems, SCM optical systems, fiber nonlinearity, and fiber optical parametric amplifiers.

Mr. Wong was a Recipient of the Optical Society of America (OSA) New Focus Student Award in 2003. He has received the IEEE/LEOS Graduate Student Fellowship. He is a Reviewer for IEEE Photonics Technology Letters.

Leonid G. Kazovsky (M'80-SM'83-F'91) was with Bellcore (now Telcordia) doing research on WDM, high-speed and coherent optical fiber communication systems. Since 1990, he has been Professor of Electrical Engineering at Stanford University. After joining Stanford, he founded Optical Communication Research Laboratory (OCRL). He has led OCRL since then. While on Bellcore assignments or Stanford sabbaticals, he has worked at the Heinrich Hertz Institute, Berlin, Germany; Hewlett-Packard Research Laboratories, Bristol, U.K.; and the Technical University of Eindhoven, Eindhoven, The Netherlands. Through research contracts, consulting engagements, and other arrangements, he worked with many industrial companies and U.S. government agencies including Sprint, DEC, GTE, AT\&T, IVP, Lucent, Hitachi, KDD, Furukawa, Fujitsu, Optivision, and Perimeter on the industrial side and the National Science Foundation, the Defense Advanced Research Projects Agency, the Air Force, the Navy, the Army, and BMDO on the government side. In 1998-1999, he took a one-year leave from Stanford University and launched a startup company known as Alidian Networks. He has served as a Reviewer for journals such as Wireless Networks; funding agencies (the National Science Foundation, OFC, ERC, the National Research Council, etc.); and publishers (Wiley, Macmillan, etc.). He has authored or coauthored two books, some 150 journal technical papers, and a similar number of conference papers.

Prof. Kazovsky is a Fellow of the Optical Society of America. He serves or has served on Editorial Boards of IEEE TRANSACTIONS ON COMMUNICATIONS and IEEE Photonics TeChNOLOgY LeTters and on Program Committees of leading conferences (OFC, the Conference of Lasers and Electro-Optics, the Laser and Electro-Optics Society, the International Society for Optical Engineers, and the Global Communications Conference). He has also served as a Reviewer for various IEEE and IEE transactions, proceedings, and journals. 